\title{
Reduction of cogging torque of single phase brushless DC motor using flux barrier
}

\begin{abstract}
This paper deals with the reduction of cogging torque in a single phase brushless DC motor. The constructional features and operating principles of the developed motor is presented. Reduction of the cogging torque is achieved by the use of slit as flux barrier. By using slit to stable the flux density of the motor, the cogging torque produced by the motor is reduced. Simulation studies to evaluate the aspects of the flux distribution inside the machine and the cogging torque characteristic are presented.
\end{abstract}

Keyword: Cogging torque; Flux barrier; Slit 